\title{
Editorial: How to respond to reviewers and revise your article for publication
}

When you submit a paper for consideration in Women and Birth, it is may be rejected by the Editor $(\sim 70 \%)$ or sent to an Associate Editor to manage the peer review process ( $30 \%)$. Article rejection is outside the scope of this Editorial, but clearly addressed by Likkis and Swett (2019). ${ }^{1}$ If the Editor invites authors to revise their submission, it is essential to submit a formal response which demonstrates appropriate revision or rebuttal in response to the reviewer's comments.

\section{Step 1 Read and reflect on the feedback}

Before you begin a response, take your time reading and thinking about the reviewers' comments. It is not uncommon to have an emotional reaction to suggested weaknesses or changes. ${ }^{2}$ Discussing the comments and your thoughts with your co-authors and/or a trusted mentor can assist you to understand them and process how to respond. ${ }^{3}$ You may be frustrated that the reviewers seem to misunderstand your meaning, or that they have missed key points in your manuscript. ${ }^{3}$ Sometimes drafting an early version of a response, including your emotional reactions, can help vent frustration or opposition to a comment. ${ }^{2}$

\section{Step 2 Start with a 'down draft'}

The first step to writing your formal response is to get them down on paper in a brainstorming way. ${ }^{4}$ The easiest way to present your response is to create a table (see example provided by Table 1). As you paste reviewer comments into the first column, it can help to transform them into a numbered list. If a reviewer has made multiple points within one sentence or comment, separate the points allocating one row to each. The down draft might still include questions or indicate areas where you are unsure how to respond; try and brainstorm some possibilities here.

\section{Step 3. Revise responses to produce an 'up draft'}

This step is so named because you can now refine and 'fix up' your initial draft. ${ }^{4}$ Make sure the tone is respectful and polite. For example, if a reviewer did not understand something, apologise for not making the point clearly enough. ${ }^{2}$ If a reviewer demonstrates that they are not an expert in the topic, remember your goal is to make the work accessible to all readers, not just experts. ${ }^{2}$ Aim to show that you have taken each comment seriously and made the asked for changes wherever possible. ${ }^{2}$ Begin each response with a direct statement such as "we agree", then expand on how and where you made the change (such as line numbers or section title). ${ }^{2}$ Effective responses will consist of clear, concise statements replying to every comment and specifying where in the manuscript changes have been made; ${ }^{3}$ see Table 1 for examples.

Sometimes you might disagree with a comment. General guidance is to make the suggested change if possible, to ensure the reviewer feels their feedback was listened to. ${ }^{2}$ Alternatively, you do not need to make changes if there is a clear rationale not to do so. However, you do need to clearly and politely rebut the suggestion, using evidence to support your argument. Table 1 (Reviewer 2, Point 4) demonstrates that authors did not necessarily do exactly as requested by the reviewer, but they explained their rationale and still revised the manuscript in a way which was acceptable to reviewers.

\section{Step 4. Complete your 'dental draft'}

This step involves a line-by-line check ${ }^{4}$ to ensure all comments have been effectively addressed. You also need to do a careful cross-check between the changes listed in the Response to Reviewer's Table, and 
the actual changes made in the manuscript (e.g. check line numbers correspond). Changes can be made with de-identified track changes or by coloured font or highlights. Whichever you use, just make sure it is quick and easy for reviewers to see where and how comments have been addressed in the manuscript. ${ }^{3}$ When checking your rebuttal points, ask yourself whether you have provided a clear rationale, whether you have provided evidence to support your argument, and whether you can make even a minor change that might help to address the concern (along with rebuttal against major changes).

\section{Step 5. Cover letter}

Provide an overview of major changes and communicate to the Editor that you have taken the feedback seriously. ${ }^{3}$ You might like to express gratitude for the work the reviewers did to provide feedback on your manuscript; and broadly identify how the manuscript has improved as a result of their time. ${ }^{5}$

\section{Step 6. Submission}

The minimum required for submission will be: 1 ) cover letter; 2) table of response to reviewers; 3 ) revised manuscript with track changes or highlights; and 4) 'clean' version of your manuscript (this is optional for Women and Birth). ${ }^{5}$

Responding to reviewer feedback and revising your article is a normal part of the peer review process. We hope these six steps will assist you to strengthen your paper and secure an acceptance for publication.

\section{References}

1. Likis FE, Swett B. Demystifying the journal submission, peer review, and publication process. J Midwifery Womens Health. 2019; 64(2):145-8.

2. Noble WS. Ten simple rules for writing a response to reviewers (Editorial). PLoS Comput Biol. 2017; 13(10):e1005730-e.

3. Hiemstra PS. How to write a response to the reviewers of your manuscript. Breathe (Sheff). 2018; 14(4):319-21.

4. Lamott A. Shitty first drafts. In: Eschholz P, Rosa A, Clark V, editors. Language awareness: readings for college writers. $9^{\text {th }}$ ed. Boston: Bedford/St. Martin's; 2005. p. 93-6.

5. Carnovale C. How to respond to reviewer comments - the CALM way. Elsevier: Authors' Update. 2019. Available from: https://www.elsevier.com/connect/authors-update/how-to-respond-toreviewer-comments-the-calm-way 
\begin{tabular}{|l|l|} 
1. Lack of reminders is one of the most important limitations of this study. & Yes, this is an interesting point. As this was an anonymous, online survey, there was
\end{tabular} How did you manage this problem?

no recruitment of participants including participant contact details. This made it

impossible to send a 'reminder' to potential participants, which has now been addressed in Limitations (track changes: Lines 427-429).

2. How did you combine the results of the 4 variables to create the primary outcome?

We appreciate this was not as clear as it could be. Therefore Methods / Primary

outcome paragraph has been revised to provide greater clarity around the composite outcome (track changes: Lines 167-183).

Reviewer \#2 Response / Revision

\begin{tabular}{|l|l}
\hline 1. No reporting guidelines seem to be used for this manuscript. & Thank-you. The authors had used the CHERRIES checklist in preparation of the
\end{tabular} manuscript. However, in response to this comment we have now cross-checked against this guideline and included further information with respect to survey administration and recruitment processes (track changes: Lines 146-150; 155-156).

2. The aims of the study are not clearly described. Two research questions are described at the end of the introduction (page 4), but no aims are explicitly described in the manuscript (except for the abstract).

We agree this was not clear enough. The questions have been revised to read as aims, which aligns with the abstract, and now the conclusion (track changes: Lines 8185).

3. In terms of recruitment and sampling (page 5), it is unclear how the surveys were distributed. I would suggest providing more information about this topic.

Thank-you, we have now provided more detail about the types of dissemination pathways in Recruitment and Sampling (track changes: Lines 143-150)

4. Enhancing the discussion on potential source of bias would add quality to the manuscript, e.g. selection bias and recall bias.

Thank-you, selection bias has been specifically highlighted and addressed in the Strengths and Limitations section of the manuscript (track changes: Lines 431-439).

While there is potential for recall bias, women were only eligible to participate if they had given birth in the previous 3 years. One study has found women still remember their childbirth experience clearly after five years. This justification and reference have been added to Strengths and Limitations (track changes: Lines 425-428).

5. Suggest to move recommendations (from the conclusion session) to the discussion. Conclusion should be shorter and address the aims. Implications for practice (track changes: Lines 405-413). Conclusion revised to address the study aims - incidence of Pronurturance and factors associated with it (track changes: 451-455). 